\title{
Author Correction: CAR-T cells targeting a nucleophosmin neoepitope exhibit potent specific activity in mouse models of acute myeloid leukaemia
}

Guozhu Xie, Nikola A. Ivica (D), Bin Jia, Yingzhong Li (iD, Han Dong, Yong Liang, Douglas Brown, Rizwan Romee and Jianzhu Chen (D)

Correction to: Nature Biomedical Engineering https://doi.org/10.1038/s41551-020-00625-5, published online 12 October 2020.

In the version of this Article originally published, the name of the author Rizwan Romee was mistakenly written as 'Romee Rizwan'. Also, in Fig. 1b, the antigen labels in rounds 1, 3, 4 and 6 were incorrect; these rounds all used 'AIQ-HLA-A2', not 'AIO-HLA-A2'. Finally, in Fig. 3d, the $y$-axis labels for the middle and bottom rows of graphs should have read 'SLL-HLA-A2' and 'HLA-A2', respectively, rather than 'AIQ-HLA-A2'. These errors have all been corrected.

Published online: 16 December 2020

https://doi.org/10.1038/s41551-020-00677-7

(C) The Author(s), under exclusive licence to Springer Nature Limited 2020 